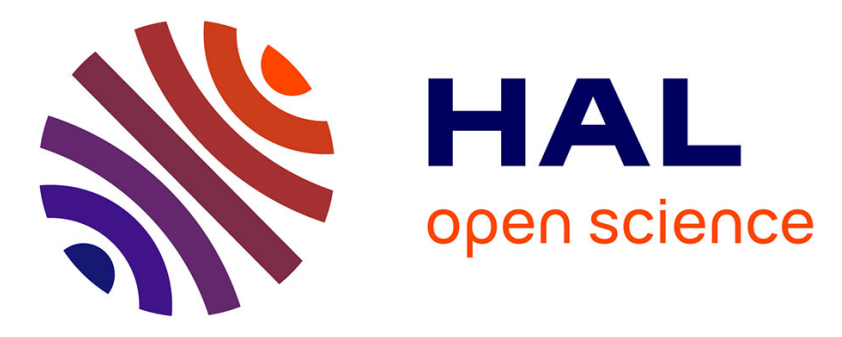

\title{
The microscopic structure of cold aqueous methanol mixtures
}

\author{
Martina Požar, Ariadni Kerasidou, Bernarda Lovrinčević, Larisa Zoranić, \\ Marijana Mijaković, Tomislav Primorac, Franjo Sokolić, Victor Teboul, \\ Aurélien Perera
}

\section{To cite this version:}

Martina Požar, Ariadni Kerasidou, Bernarda Lovrinčević, Larisa Zoranić, Marijana Mijaković, et al.. The microscopic structure of cold aqueous methanol mixtures. Journal of Chemical Physics, 2016, 145 (14), pp.144502 - 134703. 10.1063/1.4964487 . hal-01383420

\section{HAL Id: hal-01383420 \\ https://hal.sorbonne-universite.fr/hal-01383420}

Submitted on 18 Oct 2016

HAL is a multi-disciplinary open access archive for the deposit and dissemination of scientific research documents, whether they are published or not. The documents may come from teaching and research institutions in France or abroad, or from public or private research centers.
L'archive ouverte pluridisciplinaire HAL, est destinée au dépôt et à la diffusion de documents scientifiques de niveau recherche, publiés ou non, émanant des établissements d'enseignement et de recherche français ou étrangers, des laboratoires publics ou privés. 


\section{AlP $\mid \begin{aligned} & \text { The Journal of } \\ & \text { Chemical Physics }\end{aligned}$}

\section{The microscopic structure of cold aqueous methanol mixtures}

Martina Požar, Ariadni Kerasidou, Bernarda Lovrinčević, Larisa Zoranić, Marijana Mijaković, Tomislav

Primorac, Franjo Sokolić, Victor Teboul, and Aurélien Perera

Citation: The Journal of Chemical Physics 145, 144502 (2016); doi: 10.1063/1.4964487

View online: http://dx.doi.org/10.1063/1.4964487

View Table of Contents: http://scitation.aip.org/content/aip/journal/jcp/145/14?ver=pdfcov

Published by the AIP Publishing

\section{Articles you may be interested in}

Hydrogen bonded structure, polarity, molecular motion and frequency fluctuations at liquid-vapor interface of a water-methanol mixture: An ab initio molecular dynamics study

J. Chem. Phys. 141, 134703 (2014); 10.1063/1.4896233

Thermodynamical and structural properties of binary mixtures of imidazolium chloride ionic liquids and alcohols from molecular simulation

J. Chem. Phys. 129, 144503 (2008); 10.1063/1.2990653

Interfacial structures of methanol:water mixtures at a hydrophobic interface probed by sum-frequency vibrational spectroscopy

J. Chem. Phys. 125, 144711 (2006); 10.1063/1.2354088

Local structure of a phase-separating binary mixture in a mesoporous glass matrix studied by small-angle neutron scattering

J. Chem. Phys. 122, 244718 (2005); 10.1063/1.1931528

Microscopic solvent structure of supercritical carbon dioxide and its mixtures with methanol in the cybotactic region of the solute molecule

J. Chem. Phys. 108, 3915 (1998); 10.1063/1.475794

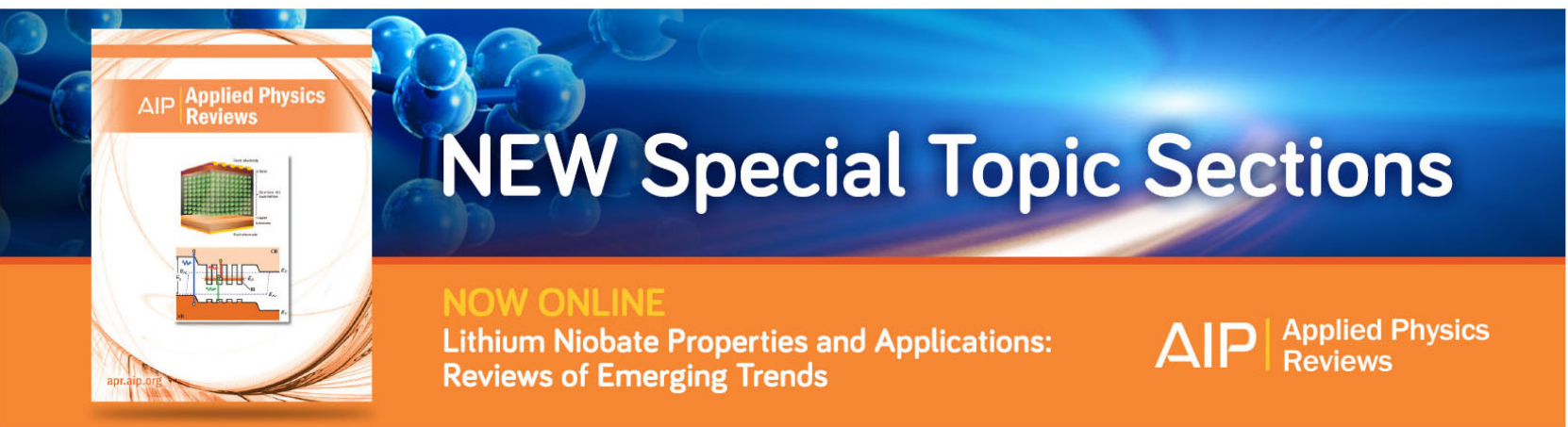




\title{
The microscopic structure of cold aqueous methanol mixtures
}

\author{
Martina Požar, ${ }^{1,2}$ Ariadni Kerasidou, ${ }^{3}$ Bernarda Lovrinčević, ${ }^{2}$ Larisa Zoranić, ${ }^{2}$ \\ Marijana Mijaković, ${ }^{2}$ Tomislav Primorac, ${ }^{2}$ Franjo Sokolić, ${ }^{2}$ Victor Teboul, ${ }^{3}$ \\ and Aurélien Perera ${ }^{1, a)}$ \\ ${ }^{1}$ Laboratoire de Physique Théorique de la Matière Condensée (UMR CNRS 7600), \\ Université Pierre et Marie Curie, 4 Place Jussieu, F75252 Paris cedex 05, France \\ ${ }^{2}$ Department of Physics, Faculty of Sciences, University of Split, Ruđera Boškovića 37, 21000 Split, Croatia \\ ${ }^{3}$ Département de Physique de l'Université d'Angers, 2 Bd Lavoisier, 49045 Angers, France
}

(Received 20 June 2016; accepted 26 September 2016; published online 13 October 2016)

\begin{abstract}
The evolution of the micro-segregated structure of aqueous methanol mixtures, in the temperature range $300 \mathrm{~K}-120 \mathrm{~K}$, is studied with computer simulations, from the static structural point of view. The structural heterogeneity of water is reinforced at lower temperatures, as witnessed by a pre-peak in the oxygen-oxygen structure factor. Water tends to form predominantly chain-like clusters at lower temperatures and smaller concentrations. Methanol domains have essentially the same chain-like cluster structure as the pure liquid at high concentrations and becomes monomeric at smaller ones. Concentration fluctuations decrease with temperature, leading to quasi-ideal Kirkwood-Buff integrals, despite the enhanced molecular interactions, which we interpret as the signature of non-interacting segregated water and methanol clusters. This study throws a new light on the nature of the micro-heterogeneous structure of this mixture: the domain segregation is essentially based on the appearance of linear water clusters, unlike other alcohol aqueous mixtures, such as with propanol or butanol, where the water domains are more bulky. Published by AIP Publishing. [http://dx.doi.org/10.1063/1.4964487]
\end{abstract}

\section{INTRODUCTION}

The fact that mixtures involving water as an associating molecular species exhibit micro-segregation is now well acknowledged. ${ }^{1-6}$ In fact, micro-segregation is a universal property of any mixture involving at least one associating component. ${ }^{7,8}$ However, the exact status of this microheterogeneity $(\mathrm{MH})$, as well as the consequences of its existence upon thermophysical properties of such systems, is not well understood. ${ }^{8,9}$ One point that we have investigated in our previous works ${ }^{8}$ is how to distinguish the microheterogeneity $(\mathrm{MH})$ from concentration fluctuations $(\mathrm{CF})$, the latter which are accessible through the Kirkwood-Buff integrals $^{10-12}(\mathrm{KBI})$. The difference between $\mathrm{CF}$ and $\mathrm{MH}$ relates, respectively, to the $k=0$ part of the structure factors (associated to the $\mathrm{KBI}$ ) and the $k \neq 0$ part corresponding to the pre-peak in partial structure factors associated with hydrogen bonding atoms. ${ }^{7}$ This latter pre-peak is associated to microsegregated domains which form through the self-hydrogen bonding of specific molecules, such as water or alcohols, for example. Indeed, mixtures of non-bonding molecules never show this characteristic pre-peak, ${ }^{7}$ and consequently are never micro-segregated, while they can perfectly well have high concentration fluctuations, which would correspond to a raise in the $k=0$ part of the structure factors. ${ }^{7}$ In our previous works on mixtures involving hydrogen bonding species, we have studied the interplay between the KBI (or equivalently $\mathrm{CF}$ ) and the domain segregation, in particular to explain the anomalous raise of the $\mathrm{KBI}^{7,8}$

\footnotetext{
a) Author to whom correspondence should be addressed. Electronic mail: aup@1ptmc.jussieu.fr
}

So far however, we have never investigated the temperature dependence of this micro-segregation. Such investigation could help clarify questions about how microheterogeneity affects the stability of a mixture. Indeed, mixtures with an upper critical solution temperature (UCST) demix when the temperature is lowered below this critical temperature. This is the case of few aqueous mixtures, such as aqueous acetonitrile ${ }^{13}$ or aqueous 2-butoxyethanol, ${ }^{14}$ for example. Aqueous acetone mixtures are known to be difficult to mix within computer simulations, ${ }^{15-17}$ and one could conjecture the existence of a hidden UCST at temperatures lower than ambient. ${ }^{15}$ Aqueous methanol solution does not undergo demixing at any temperatures for which they are liquid. However, the question of the stability remains posed, in particular if the concentration fluctuations increase or decrease with the decrease of the temperature. Similarly, one can ask how does the micro-heterogeneity evolve with decreasing temperatures? Such questions could help clarify the connections between $\mathrm{MH}$ and $\mathrm{CF}$. We can take advantage of the fact that computer simulations of aqueous mixtures are notoriously known to have difficulties to produce a crystalline phase below the experimental freezing point, ${ }^{18,19}$ to study these systems beyond the experimental conditions where they stay liquid. These difficulties are due to the problems of forming a hydrogen bonded nuclei with proper crystalline symmetry without the help of particular sampling techniques ${ }^{19}$ or models. ${ }^{20,21}$ Since we do not use such techniques here, we obtain supercooled and glassy phases at temperatures as low as $T=100 \mathrm{~K}$. From the experimental point of view, under ambient conditions, methanol crystallizes at $T=175 \mathrm{~K},{ }^{22}$ but amorphous phases can be obtained as low as $T=90 \mathrm{~K}^{23}$ 
It is then interesting to observe how $\mathrm{CF}$ and $\mathrm{MH}$ evolve in such cold mixtures. It is now well known that low temperature liquids, in the supercooled phase and beyond in the glass phase, are characterised by dynamical heterogeneity. ${ }^{24-30}$ This type of heterogeneity is characterised by the existence of spatial domains where molecules of high mobility are aggregated. It is therefore interesting to see how the existence of microheterogeneity correlates with the concomitant existence of dynamical heterogeneity, specially in a mixture where species have different mobilities.

In order to study these various problems, we have chosen aqueous methanol mixtures, namely, the first system in which micro-heterogeneity was revealed by the Soper group as an unexpected feature in a macroscopically homogeneous system. ${ }^{1}$ The same group has previously investigated this particular mixture under cooling and high pressure, ${ }^{31}$ both by EPSR techniques and computer simulations. Their analysis led them to conclude that a UCST could be hidden below the freezing line. Although the structural findings we report in this paper are quite similar to those of these authors, our analysis of the concentration fluctuations leads us to conclude at a enhancement and stabilisation of segregation at the detriment of critical concentration fluctuations. We shall discuss the pertinence of our findings in Sec. IV. The dynamical properties of this particular system in the supercooled regime were very recently investigated experimentally by Mallamace et al., ${ }^{32}$ with a particular accent over relaxation processes. Our approach is more focused on static properties and local heterogeneity.

The study of low temperature behaviour of aqueous methanol has another interest, related to the low temperature behaviour of each of the pure components, and principally water, because of the much debated existence of a second critical point. ${ }^{33-36}$ Although water is a hydrogen bonded liquid, it does not exhibit any of the specific clustering properties of methanol and higher linear alcohols. ${ }^{37,38}$ Methanol is known to form chain-like linear clusters., , $37,39-41$ Under mixing, water has also the propensity of forming linear chain-like clusters, such as in aqueous dimethyl sulfoxide (DMSO) mixtures. ${ }^{8,42}$ It is therefore interesting to ask if such type of clustering has any relation with the existence of a liquidliquid phase separation ${ }^{36,43}$ at low temperatures. This type of problem can be clarified by examining the relation between concentration fluctuations-related to phase transitions, and micro-heterogeneity.

The remainder of this manuscript is as follows. In Sec. II we describe briefly the difference between $\mathrm{CF}$ and $\mathrm{MH}$, and we provide information on our computer simulations. In Sec. III we discuss our findings for static structural and dynamical properties. Sec. IV gathers the discussion about the principal features unveiled in Sec. III as well as our conclusions and perspectives.

\section{THEORETICAL AND COMPUTATIONAL DETAILS}

\section{A. Concentration fluctuations and micro-heterogeneity}

In a many component mixture, the macroscopic concentration fluctuations are defined as ${ }^{10,44}$

$$
\left\langle N_{a} N_{b}\right\rangle-\left\langle N_{a}\right\rangle\left\langle N_{b}\right\rangle,
$$

where $a$ and $b$ are species index, $N_{\alpha}$ is the number of particles of species $\alpha$ in a volume $V$, and the bracket indicate the grand canonical ensemble average. ${ }^{44}$

As emphasized in our previous works, ${ }^{7,8,45,46}$ these concentration fluctuations are related to the $k=0$ part of the structure factors through the relation

$S_{a b}(k=0)=\frac{\left\langle N_{a} N_{b}\right\rangle-\left\langle N_{a}\right\rangle\left\langle N_{b}\right\rangle}{\sqrt{\left\langle N_{a}\right\rangle\left\langle N_{b}\right\rangle}}=\frac{V}{\sqrt{N_{a} N_{b}}}\left(\frac{\partial \rho_{a}}{\partial \beta \mu_{b}}\right)_{T V \mu_{c}}$,

where $a$ and $b$ are species index, and where the $\rho_{\alpha}=N_{\alpha} / V$ and $\mu_{\alpha}$ represent the number of particles, the partial number density, and the chemical potential for species $\alpha$, respectively, with $V$ being the total volume and $\beta=1 / k_{B} T$ being the Boltzmann factor (with $k_{B}$ being the Boltzmann constant and $T$ the absolute temperature). The structure factor $S_{a b}(k)$ is related to the Fourier transform of the pair distribution function $g_{a b}(r)$ through the expression ${ }^{7}$

$S_{a b}(k)=\delta_{a b}+\frac{1}{V} \sqrt{N_{a} N_{b}} \int d \vec{r}\left[g_{a b}(r)-1\right] \exp (i \vec{k} . \vec{r})$.

Eq. (2) holds equally for molecules made of various atoms. This is because all the atom-atom structure factors $S_{i j}(k)$ for a single species (with atoms $i$ and $j$ ) have the same $k=0$ value, which is also that of the molecular center of mass invariance of the integral in Eq. (3) for $\mathrm{k}=0$, with respect to the choice of the origin. ${ }^{44}$

In contrast to Eq. (2), where concentration fluctuations acquire clear physical significance, micro-heterogeneity, which can be seen as a wave-vector dependent fluctuation, cannot be directly linked to any measurable quantities such as in Eq. (2), which has made its detection problematic. However, MH has a well-defined signature in terms of a pre-peak in specific atom-atom structure factors, as we have previously shown. ${ }^{45-48}$ This pre-peak arises in different context which we have encountered in our previous investigations. It could be due to the presence of specific micro-segregated domains, such as those found in aqueous-alcohol ${ }^{47,48}$ or alkane-alcohol ${ }^{7}$ mixtures. It could be due to the presence of specific clusters, such in neat alcohols, ${ }^{37,39,53}$ or in mixtures such as aqueous$\mathrm{DMSO},{ }^{42}$ both in which chain-like cluster of the hydroxyl groups is observed.

Concentration fluctuations in mixtures can be appreciated through the Kirkwood-Buff integrals, ${ }^{10,11}$ which are defined by the expression

$$
G_{a b}=\int d \vec{r}\left[g_{a b}(r)-1\right]
$$

which can be equally computed through the atom-atom pair distribution functions $g_{i j}(r)$ for the same reason as mentioned above. In practice, some care should be taken when evaluating these integrals through site-site functions obtained by computer simulations, since the presence of concentration fluctuations tends to shift the asymptote of these functions to a value different than $1 .^{7}$ When mixtures behave in a near ideal manner, their concentration fluctuations are small, and this correction can be avoided. This was the case here for very low temperatures. The KBI for binary mixtures can 
be expressed in term of various thermodynamical quantities as $^{11,12}$

$G_{a b}=\left(1-\delta_{a b}\right)\left(\kappa_{T}^{*}-\frac{\bar{V}_{1} \bar{V}_{2}}{V D}\right)+\delta_{a b}\left[G_{12}+\frac{1}{x_{a}}\left(\frac{\bar{V}_{c}}{D}-V\right)\right]$,

where $\kappa_{T}^{*}=(1 / \rho)(\partial \rho / \partial \beta P)_{T}$ is the reduced compressibility (which can be neglected for dense liquids), $\bar{V}_{a}$ and $x_{a}$ are the partial molar volume and mole fraction of species a, respectively, $V$ is the molar volume, and

$$
D=x_{1}\left(\frac{\partial \beta \mu_{1}}{\partial x_{1}}\right)=x_{2}\left(\frac{\partial \beta \mu_{2}}{\partial x_{2}}\right) \text {. }
$$

By separating the chemical potential into the three usual parts, ${ }^{44}$ which are reference, ideal, and excess parts,

$$
\mu_{\alpha}=\mu_{\alpha}^{(0)}+\mu_{\alpha}^{\text {ideal }}+\mu_{\alpha}^{\text {excess }},
$$

where $\mu_{\alpha}^{(0)}=k_{B} T \ln \Lambda^{-3}$ (with $\Lambda$ being the de Broglie thermal wavelength, which depends only on the mass of the particle ${ }^{44}$ ) and $\mu_{\alpha}^{\text {ideal }}=k_{B} T \ln \rho_{\alpha}$, we see that an interesting case occurs when one has $D=1$, which implies from Eq. (6) that $\mu_{1}^{\text {excess }}=\mu_{2}^{\text {excess }}=0$, conditions which can be achieved in the absence of interactions. The denomination of "ideal mixture" is associated to the case $D=1$. In fact, $D=1$, or equivalently $\mu_{a}^{\text {excess }}=0$, do not necessarily imply the absence of interactions, since the excess chemical potentials can be zero when the entropy and enthalpy contributions cancel each other. This is postulated to happen in the case when some local ordering of the molecules is present, and for which entropic and enthalpic contributions cancel each other. $^{49,50}$ The appearance of micro-heterogeneous domains could correspond to such local molecular ordering, which could therefore produce a "fake" ideality with $D \approx 1$. We will illustrate below such a case in the very low temperature cases.

\section{B. Computational details}

All simulations were conducted with the Gromacs 4.6 package. ${ }^{51}$ We mostly used system sizes of $N=2048$ molecules, which corresponds on average to box sizes about $45 \AA$. The box size change from $T=300 \mathrm{~K}$ to $T=200 \mathrm{~K}$ is $3 \%$, which corresponds to a very small volume change. For example, for pure methanol, we find that the molar volume varies from $V_{m}=40 \mathrm{~cm}^{3} / \mathrm{mol}$ at $T=300 \mathrm{~K}$ to $38 \mathrm{~cm}^{3} / \mathrm{mol}$ at $T=200 \mathrm{~K}$ and $36 \mathrm{~cm}^{3} / \mathrm{mol}$ at $T=100 \mathrm{~K}$. Static properties were calculated in the isothermal isobaric (constant NPT) ensemble. The temperature was maintained constant by a modified Berendsen thermostat and pressure maintained constant with the Parrinello-Rahman barostat (both with time constant of $0.1 \mathrm{ps}$ ). The leap-frog integrator time step was fixed at $1 \mathrm{fs}$. We followed the same protocol for all of our simulations. The initial configurations were prepared with the package Packmol ${ }^{52}$ with the molecules put in a box size computed by interpolating linearly the experimental volumes of the pure components. This way, we minimize the equilibration part, by starting with an appropriate initial volume. Static properties were computed with 2-4 ns statistics. Typically, we studied cases with the methanol mole fractions $x=0$ (pure water), $x=0.2$, $x=0.5, x=0.8$, and $x=1$ (pure methanol). The pure components were studied essentially for dynamical properties, since we have reported static properties earlier. ${ }^{37,54,56}$ The static correlation functions were obtained typically over 2000 independent configurations. The site-site structure factor reported here is calculated by direct Fourier transform of the site-site correlation functions by standard numerical methods. ${ }^{57}$

The SPC/E water model ${ }^{58}$ was used and the OPLS model $^{59}$ and TraPPe model ${ }^{60}$ for methanol. There is very little differences between these 2 models in the mixing conditions. The OPLS model tends to show enhanced concentration fluctuations when compared to the TraPPe model, visible through the higher $S(k=0)$ values. However, both structure factors are nearly identical at higher k-values. These observations imply that thermo-physical propertiesessentially obtained at $k=0$, could show some model dependence, while micro-heterogeneity-sampled at $k \neq 0$ might be model independent.

We studied very low temperatures, well below freezing temperatures. However, our system remained liquid or supercooled/glassy since it is very hard to obtain crystallisation of aqueous mixtures without specific manipulations and models. ${ }^{19}$ One important point about super-cooled glassy states is that these are clearly non-equilibrium or metastable states. Therefore one could ask how one can apply equilibrium thermodynamic criteria and compute or measure equilibrium quantities. This question is relevant to our study since we report here many equilibrium quantities such as the distribution functions and the Kirkwood-Buff integrals. This important point has been answered many times in the vast literature of the supercooled/glassy states. ${ }^{61,62}$ The main argument line is the following. Contrary to stable states which have only a single minima in the phase space, these metastable states have many minima. These minima are replicas of each other, and the glass transition is seen as a replica symmetry breaking, ${ }^{63}$ which splits equivalent equilibrium minimum into distinct ones. The system gets trapped in any one of these minima for very long times, and can go to another only through an activated transition. ${ }^{27,65}$ While the system is trapped inside a single minimum, equilibrium criteria apply and one can compute and measure a variety of them. ${ }^{62,64,65}$ This situation happens equally in computer simulations. ${ }^{29}$

The computation of the structure factors and KirkwoodBuff integrals through correlation functions evaluated in computer simulation is a topic we have addressed in previous papers (see, for example, Ref. 7), which we briefly recall here. The principal problem with correlation functions in computer simulations is that they do not decay to unity,

$$
\lim _{r \rightarrow \infty} g_{a b}(r)=1-\frac{\varepsilon_{a b}}{N},
$$

where $N$ is the number of particles in the box, and the coefficient $\varepsilon_{a b}$ is related to the concentration fluctuations in Eq. (2) and this depends also on the type of statistical ensemble used to do the simulations. ${ }^{7}$ As a consequence, 
the expression in Eq. (4) cannot be used to compute the Kirkwood-Buff integrals. However, since the asymptote of the $g_{a b}(r)$ nevertheless exists, we have devised a simple methodology to remove this value-instead of 1, in Eq. (4). This has been illustrated in several of our previous works, and Kirkwood-Buff integrals evaluated for several types of systems, and often in good agreement with experimental values. ${ }^{7,42,47,48,54}$ In particular, the $\mathrm{KBI}$ of the $\mathrm{T}=300 \mathrm{~K}$ aqueous-methanol mixture have been evaluated in Ref. 54 . In the present case of lower temperatures, the correlation functions tend to have longer ranged oscillations instead of decaying to a value close to 1 . However, such oscillations are very small, especially for the system size of $\mathrm{N}=2048$ molecules. Consequently, the value very close to $k=0$, in the range $0<k<1.5 \times 2 \pi / L \approx 0.18 \AA^{-1}$ (where $\mathrm{L}$ is the box size, often around $L \approx 47 \AA$ for $\mathrm{N}=2048$ ), tends to be biased slightly. However, the structure factor values in the range $\mathrm{k}>0.5$ are totally insensitive to the box size. We confirmed this by simulating few systems with twice larger (with $\mathrm{N}=16000$ ): only the structure factor values below $k<0.25 \AA^{-1}$ are affected by system size. In these conditions, the KBI cannot be evaluated safely from the $\mathrm{k}=0$ values of the structure factors, according to Eqs. (2)-(4). Instead, we use quantities that we called "running KBI" (rKBI) in our previous works, ${ }^{47,54,56}$ defined as

$$
G_{a b}(R)=4 \pi \int_{0}^{R} d r r^{2}\left[g_{a b}(r)-1\right] .
$$

Obviously, this function tends asymptotically to the KBI $G_{a b}$ when $R \rightarrow \infty$. These quantities have a flat asymptote which corresponds to the value of the KBI according to Eqs. (4) and (9), which can be evaluated graphically. In the case of domain formation, the RKBI reach the asymptote through domain oscillations, which range can exceed the simulation box size. ${ }^{47,48}$ Even then, the center of such oscillations often provides a good estimate of the KBI. In the case of aqueous mixtures, the fluctuating water domains often do not allow to obtain a flat asymptote behaviour of the RKBI. This is due to intrinsic domain fluctuations, which do not allow to stabilize the asymptote, which we have previously reported for aqueous methanol at $\mathrm{T}=300 \mathrm{~K} .{ }^{56} \mathrm{In}$ such cases, we use a trick from the fact that the solute-solute and cross correlation have often well or better defined asymptotes. We use the KBI extracted from these rKBI asymptotes to obtain the self-consistent water-water KBI from Eq. (5), where we compute $G_{w w}$ from $G_{w s}$ and $G_{s s}$ by eliminating the D coefficient. We check afterwards the consistency of this value with the water-water asymptote of the $G_{w w}(R)$, and this procedure always provides an excellent estimate of $G_{w w}$. These problems and approaches are illustrated in Fig. 1, where we show a comparison between the system size we have mostly used in this work, with $\mathrm{N}=2048$ particles, and twice as much with $\mathrm{N}=16384$. Both results have been obtained for 4 ns long runs. The top panel shows the water oxygen correlations and structure factor, and it is seen that the agreement is near perfect, both in real and reciprocal space, except for very small $\mathrm{k}$ values. The small pre-peak of the structure factor is reproduced for both sets of data, with a small shift for the larger system. The middle and lower panels illustrate the rKBI asymptotes for the oxygen

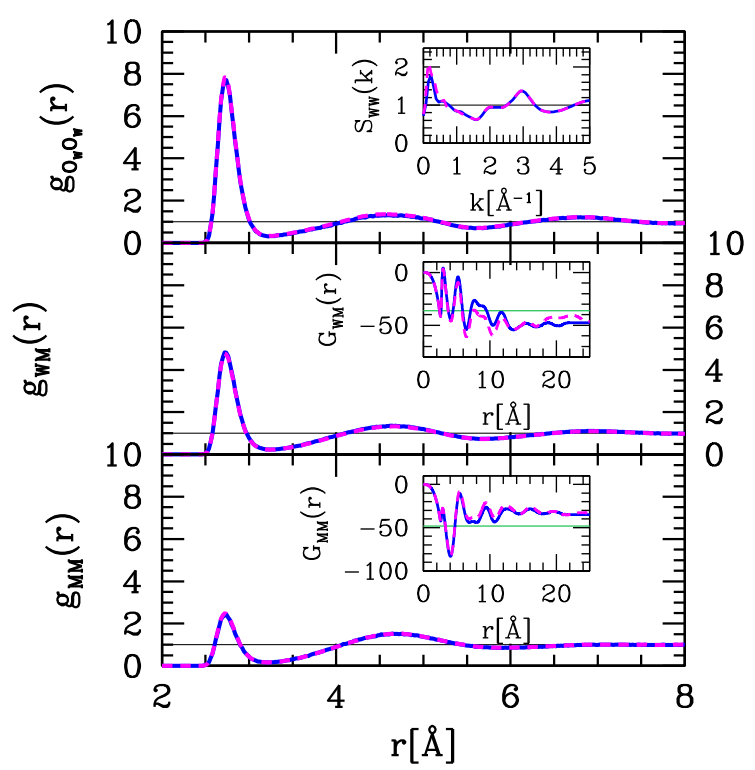

FIG. 1. Comparison of structural quantities between system sizes $\mathrm{N}=2048$ (blue) and $\mathrm{N}=16384$ (dashed magenta), for equimolar aqueous methanol at $\mathrm{T}=200 \mathrm{~K}$. (Top) Main panel: water oxygen-oxygen correlation $g_{W W}(r)$; inset: corresponding structure factors $S_{W W}(k)$. (Middle) Main panel: watermethanol oxygen atom correlations $g_{W M}(r)$; inset: corresponding rKBI functions $G_{W M}(r)$ (the green line is the ideal KBI value). (Bottom) Same as middle panel, but for methanol oxygen-oxygen correlations.

atom correlations between water and methanol (middle) and methanol (lower panel). It is seen that the rKBI do indeed go to a flat asymptote-corresponding to the KBI values-with some oscillatory structure. The flat asymptotes are obtained by shifting the initial data from computer simulation as explained in our previous papers. ${ }^{7,54}$ Omitting this correction leads to slanted asymptotes, ${ }^{7,54}$ which are obviously incorrect trends. The water-water KBI is evaluated both through direct asymptote evaluation (which is not often as accurate as the two others because of the cluster/domain dynamics that affects the statistics), or using the trick mentioned above. The green lines in the middle and lower insets represent the ideal KBI values, which we discuss in Section III D below.

\section{RESULTS AND DISCUSSION}

We first examine the temperature and concentration dependence of the static properties, related to the microscopic distribution of the particles, such as structural properties and concentration fluctuations through the KBI. Then we examine dynamical properties, particularly in the supercooled regime.

\section{A. Snapshots}

Since simple liquids are disordered systems, textbooks on computer simulations discourage looking at snapshots which are generally not indicative of the structural organisation. ${ }^{66}$ However, in the case of complex molecular organisations, snapshots are particularly interesting, and should be observed. This is, for example, the case of room temperature ionic 


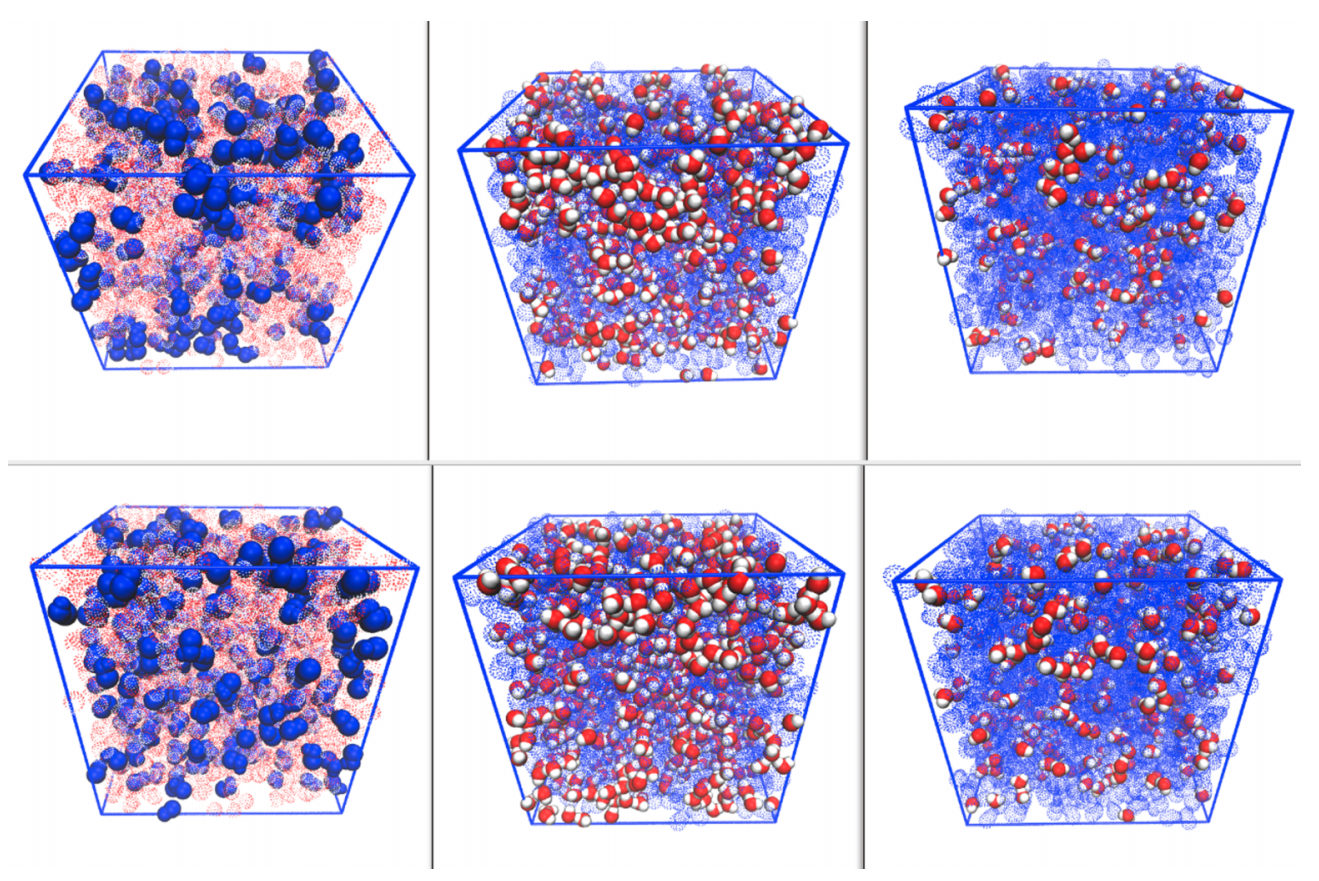

FIG. 2. Snapshots of aqueous methanol for different concentrations and two temperatures: $\mathrm{T}=300 \mathrm{~K}$ (upper row) and $\mathrm{T}=200 \mathrm{~K}$ (lower row). Leftmost, middle, and rightmost figures correspond to $20 \%, 50 \%$, and $80 \%$ of methanol, respectively. Methanol molecules are shown in dark blue, the oxygen and hydrogen of water molecules in red and white, respectively.

liquids, where the polar/apolar segregation of molecular moieties is distinctively seen through snapshots. ${ }^{67,68}$ Similarly, in micro-heterogeneous mixtures, snapshots are indicative of how this statistically permanent structure appears and evolves. ${ }^{8}$ Fig. 2 shows snapshots of the micro-heterogeneous structure of aqueous methanol mixtures at two different temperatures. The visual inspection indicates that there are more changes with concentration than with temperature. These pictures confirm that, in the absence of crystallisation, these mixtures stay micro-heterogeneous, much like at room temperature. Looking closer, one may notice a slight reinforcement of the micro-segregation by better defined domains. This will be confirmed by the analysis of the structure functions below. At low water concentrations (rightmost panels), water distinctively appears to form threadlike clusters. These clusters appear better defined at the lower temperature (bottom figure) than at the higher one (upper figure). This is equally confirmed by the structural study below.

\section{B. Radial distribution functions}

Fig. 3 gives essentially the evolution of first neighbour oxygen atom correlations with decreasing temperature. We expect these correlations to increase with decreasing temperatures, because of the subsequent increase of the Coulomb interactions, resulting in a tightening of the H-bonds in our classical description. ${ }^{69}$ This is what we see both for water and methanol, and for every concentration. However, we note striking differences between water and methanol. First of all, water oxygen correlations increase with decreasing water concentrations, while that of methanol do exactly the opposite: they decrease with decreasing methanol concentrations. This fact was previously pointed out by us for other aqueous ${ }^{15,42,48}$ and non-aqueous ${ }^{54}$ mixtures, involving at least one hydrogen bonding species: at the respective low concentrations, the lesser bonded species (or non-bonding species) has always lower correlations than the H-bonding species. This fact reflects a peculiarity of the H-bond interaction, which seems to favour the more bonding species over the less bonding ones. The second point concerns the relative increase of correlations of the first neighbours, which is more dramatic for water than for methanol. The third point concerns the second neighbour correlations, which go through a maximum at $200 \mathrm{~K}$ for water (green curves)-for all methanol concentrations, while for methanol, this happens at $150 \mathrm{~K}$ (magenta curves). This is

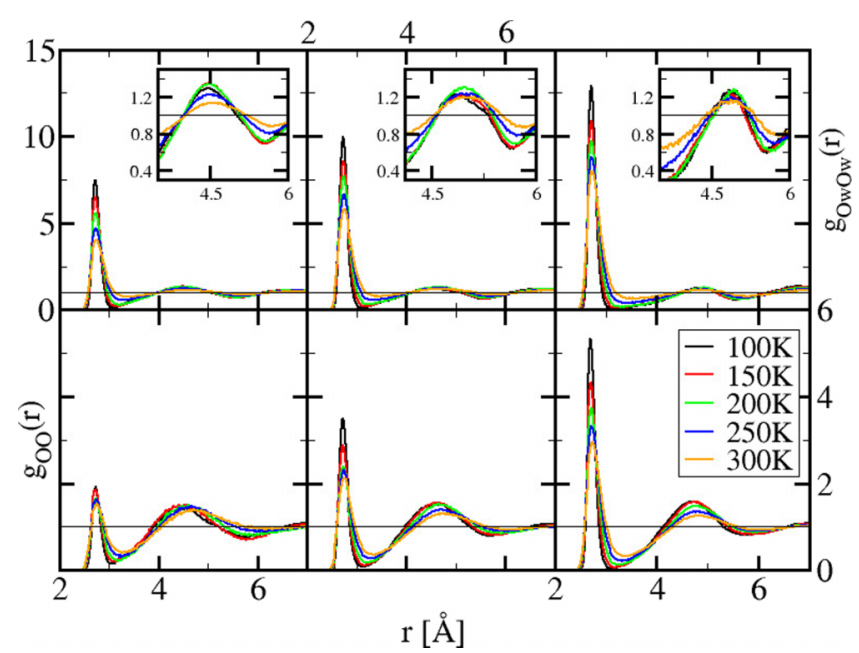

FIG. 3. Temperature dependence of the oxygen-oxygen correlation functions. Upper row for water and lower for methanol. Leftmost, middle, and rightmost figures correspond to $20 \%, 50 \%$, and $80 \%$ of methanol, respectively. The insets in the upper row figures are zoom over the second peak features. 
shown in the insets of the upper row figures of Fig. 3. This decrease of second neighbour correlations at low temperatures, while the first neighbour correlation always increase, indicates a thinning of the H-bond network, while it gets reinforced. The last point concerns the water-water correlations at second and higher neighbours, for low water concentrations (right most panels). One sees that these correlations tend to oscillate sightly below 1 , which indicates a decrease of the number of neighbours. The findings about the second neighbour correlations are quite similar to those reported previously by the Soper group. ${ }^{31}$ This confirms the predominance of linear H-bonded clusters, which we pointed out in Sec. III A above.

In order to confirm this point, we have plotted in Fig. 4 the oxygen-oxygen correlation functions together with the corresponding coordination numbers defined as

$$
n_{O_{A}}(R)=\frac{4 \pi N_{A}}{V} \int_{0}^{R} d r r^{2} g_{A A}(r)
$$

where $O_{A}$ is the oxygen atom of species $A$ (water or methanol) and $N_{A}$ is the number of particles of species $A$ in the volume $V$. This quantity should inform us on the hydrogen bonding behaviour for each species, both in temperature and methanol concentration. One sees clearly that the H-bonding coordination of water decreases below that of methanol at the highest methanol content $(80 \%$ in the right most panels), which could be at first considered as consistent with the corresponding low concentration of water. However, in addition, we observe the flattening of the water coordination, which supports the existence of the chain-like aggregates we see in the snapshots. This is more pronounced at $\mathrm{T}=200 \mathrm{~K}$ (lower right panel). Methanol coordinations, indicate no sign of particular chain formations at low concentrations, whereas one recovers the same shape as for pure methanol at the $80 \%$ methanol content, suggesting the existence of methanol chains, as observed in the snapshots.

The general information that we gather from examining these correlation functions is that methanol molecules are generally less hydrogen bonded to themselves, and more so at lower concentrations, while water molecules form clear

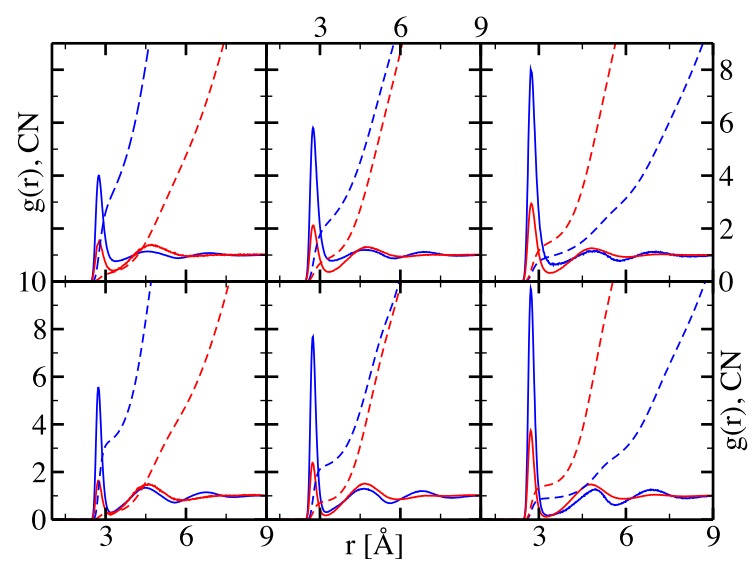

FIG. 4. Oxygen-Oxygen correlation functions (full lines) and corresponding coordination numbers (dashed lines). Water in blue and methanol in red. Top panels for $\mathrm{T}=300 \mathrm{~K}$ and lower panel for $\mathrm{T}=200 \mathrm{~K}$. Left figures for methanol concentration $x=0.2$, middle for $x=0.5$ and right for $x=0.8$. linear aggregates at low water concentrations. This is very different than other aqueous mixture of higher alcohols, where we found more bulky domains. ${ }^{8,47,48}$ The presence of such domains gives rise to long range domain oscillations in the water oxygen atom correlations, ${ }^{8,47,48}$ unlike here, where the water cluster signature is in the short range part.

\section{Structure factors}

The structure factors provide a better idea of the overall microscopic structure than the radial correlation functions, the latter which are more appropriate to study first neighbour correlations.

Fig. 5 shows somewhat an inverse global tendency than that we see in Fig. 3. Indeed, we see that it is water that shows the most remarkable features in the small-k region $0.2<k<1$. At $\mathrm{T}=300 \mathrm{~K}$, the water oxygen-oxygen structure factors show mostly concentration fluctuations, as seen from the large $\mathrm{k}=0$ raise. However, as the temperature is lowered, the CF decrease-as witnessed by the decrease of $\mathrm{k}=0$ part of the structure factor, and an intermediate broad pre-peak structure emerges, in the range $k=0.2-1 \AA^{-1}$, which has considerable inner structure, as can be seen from the small oscillatory sub-structures. This range corresponds to domains of size $6-30 \AA$. These are not numerical artifacts, and persist with long runs, and larger system size. The pre-peak feature looks more like a plateau for methanol mole fraction 0.2 (leftmost panel), and a bump for higher mole fraction (right panel). The broad pre-peak witness the water domains, which are more apparent at lower water concentrations, such as $50 \%$ and $80 \%$ methanol content. The number of substructures increases with methanol content, suggesting the persistence of particular forms of water $\mathrm{H}$-bonded aggregates, which correspond to the linear aggregates we observed in the snapshots. The main peak of water at $\mathrm{T}=300 \mathrm{~K}$ shows the peculiar double peak structure, ${ }^{55}$ with one peak at $k=2 \AA^{-1}$ which corresponds to the diameter of water $\sigma \approx 3 \AA$, and an outer-peak at $k=3 \AA^{-1}$, which corresponds to the $\mathrm{H}$ bonding $\mathrm{O}-\mathrm{O}$ distance $r_{H B} \approx 2 \AA$. This double-peak structure changes little at $50 \%$ and $80 \%$ methanol content, indicating

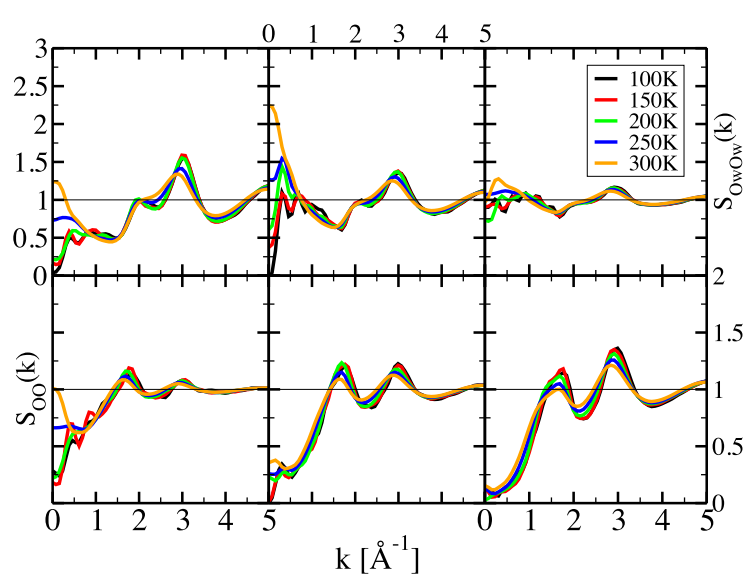

FIG. 5. Temperature dependence of the oxygen-oxygen structure factors corresponding to Fig. 3. 
the presence of strongly bound water molecules. However, this structure is enhanced at $20 \%$ methanol content, indicating that water tightens its $\mathrm{H}$-bond structure. These two features give an indirect picture of the nature of the H-bonding in the domain cluster structures which give rise to the broad pre-peak. This pre-peak structure is model independent since we could reproduce it both for other methanol and water models. We believe it must be a genuine feature of the real mixtures. We also note that the smallness of these peaks in sharp contrast with the large pre-peak we observe for aqueous mixtures of higher alcohols. ${ }^{8,47,48}$ This is another signature that these pre-peaks are not related to the bulky segregated water domain, but rather to the linear clusters. In that, the pre-peak structure found here resembles that of aqueousDMSO, where we also found that water forms linear chain clusters. $^{42}$

Examining the O-O structure factor of methanol, we recall from our previous reports ${ }^{37,70}$ that, at $\mathrm{T}=300 \mathrm{~K}$, pure methanol shows a broad pre-peak in the range $k=1-1.5 \AA^{-1}$ as well as another outer peak at H-bonding distance, just like water, at $k=3 \AA^{-1}$. The pre-peak corresponds to chain and loop H-bonded clusters of size ranging $6-4 \AA$, which correspond to the type of chains we found in our cluster analysis of pure methanol. ${ }^{37}$ Here, we see that both peaks become more accentuated with decreasing temperature.

In contrast to water, methanol $\mathrm{O}-\mathrm{O}$ structure factor shows almost no domain pre-peak structure, hinting to looser methanol domains. However, we see a considerable tightening of both the main peak at $k=3 \AA^{-1}$ and the cluster-prepeak at $k \approx 1.5 \AA^{-1}$, and at methanol concentrations $50 \%$ and $80 \%$. The $20 \%$ case is more ambiguous. We have seen from the snapshots that methanol forms very loose clusters. The structure factor at $20 \%$ shows less accentuated evolution of these 2 peaks, as well as a broad raise at $\mathrm{k}=0$, all of this suggesting indeed loose cluster structure between mere concentration fluctuations and chain structures.

\section{Kirkwood-Buff integrals}

The KBI witness the macroscopic concentration fluctuations in the system. Fig. 6 shows the temperature dependence of the KBI of the aqueous methanol mixtures for $\mathrm{T}=300 \mathrm{~K}$

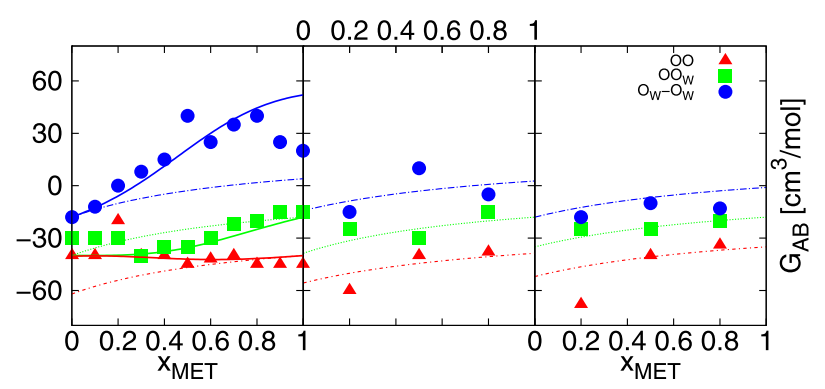

FIG. 6. Kirkwood-Buff integrals of the aqueous methanol mixtures for $\mathrm{T}$ $=300 \mathrm{~K}$ (left), $\mathrm{T}=200 \mathrm{~K}$ (middle), and $\mathrm{T}=100 \mathrm{~K}$ (right). Symbols are the $\mathrm{KBI}$ computed from computer simulations, full lines are experimental data, ${ }^{11}$ and dashed lines are ideal KBI (see text). (left), $\mathrm{T}=200 \mathrm{~K}$ (middle), and $\mathrm{T}=100 \mathrm{~K}$ (right). The KBI of the mixtures at $T=300 \mathrm{~K}$ have been reported earlier, ${ }^{54}$ and Fig. 6 indicates that there is an appreciable deviation from ideal behaviour, particularly for the water. As the temperature is diminished, we see that the KBI tend to become closer to the ideal behaviour (dashed lines, see below). This is particularly true for water. It is interesting to link this behaviour with the structural features discussed above as well as to the meaning of the ideality of the KBI as discussed in Section II A.

Perhaps the most confusing feature of the KBI is the interrelation between macroscopic concentration fluctuations at $k=0$ and the domain formation at $k \neq 0$ witnessed by a prepeak in specific site-site structure factors related to $\mathrm{H}$-bonding sites. Much remains to be understood about this interrelation. In a recent work on aqueous-DMSO, we observed that linear water clusters were linked to near-ideal KBI, as well as a weak pre-peak in the oxygen-oxygen structure factor of water. $^{8,42}$ In the present case, it would seem indeed that the linear water clusters observed at low temperatures tend to be correlated to corresponding domain cluster peaks and near-ideal KBI. In all cases reported in Fig. 6, the ideal KBI are obtained by stetting $D(x)=1$ in Eq. (5) and assuming linear volume dependence $V(x)=V_{W}(1-x)+V_{M} x$, where $\mathrm{x}$ is the methanol mole fraction and $V_{M}=18 \mathrm{~cm}^{3} / \mathrm{mol}$ and $V_{M}=40 \mathrm{~cm}^{3} / \mathrm{mol}$ are the molar volumes of pure water and methanol, respectively. As discussed in Section II A, the ideality of the KBI, and subsequently $D(x)=1$, does not mean that there are no interactions in the system, but rather that the system is reorganised such that the entropic and enthalpic contributions cancel each other. In the present case, it is clear that the organisation corresponds to the respective methanol and water clusters that we have observed. Since such organisations witness important inter-molecular interaction, the quasi-ideality could come only from the absence of domain-domain (or cluster-cluster) interactions. This explanation seems compatible with the fuzzier clusters observed at high temperature $\mathrm{T}=300 \mathrm{~K}$ and sharper ones at lower temperatures. Indeed, sharper clusters could be compared to hard bodies and would interact less that fuzzier clusters that can be compared to attracting bodies because of the water-methanol cross interactions. This way, one could explain why the KBI show increased non-ideality at higher temperatures than at lower ones, not because of the molecular interactions, but because of the cluster-cluster interactions. We note that the lowering of concentration fluctuations with temperature is clearly not in favour of the predicted UCST behaviour $^{31}$ and the associated increase of critical concentration fluctuations.

\section{E. Dynamical properties}

Individual molecules diffuse faster than clusters and aggregated domains. From this perspective, it is interesting to see how dynamical properties are changed with respect to domain formation, and how these vary with temperature. In a visual study of the dynamics, we find that aggregated domains decohere in the sub-picosecond scale, while at low temperature $T=200 \mathrm{~K}$ the decoherence time is larger, as 
expected in the supercooled region. Such visualisation shows how micro-heterogeneity persists over time and remains as an intrinsic and universal property of H-bonded systems, independently of the temperature.

\section{Diffusion constant}

Fig. 7 shows the temperature dependence of the diffusion constant, for an equimolar mixture. The diffusion constants show an Arrhenius behaviour for methanol and superArrhenius for water, as seen by the faster exponential decay of $D_{w}$. The behaviour of pure water and methanol is also shown. It is seen that, under equimolar mixing conditions, methanol diffuses slower than in the pure liquid, while it is the opposite for water. While the former behaviour is somewhat expected, the latter behaviour of water is an illustration of the preponderant role played by the $\mathrm{H}$-bond interactions for this liquid: these are stronger in pure water than in mixing conditions. It is instructive to recoup these informations with the existence of micro-heterogeneous domains. Water diffuses faster in smaller domains, while it is the opposite for methanol. Since the visual inspection of the snapshots shows that water domains are better defined that methanol domains (loose clusters), one would expect that water molecules would be trapped in such rigid domains, hence act slower than methanol molecules. Therefore, the various H-bond interactions play an important role in the mixtures, by slowing down methanol molecules, while water diffusion is enhanced, both cases when compared with their respective to neat liquid conditions.

\section{Structural heterogeneity versus dynamical heterogeneity}

The diffusion constants reported in Fig. 7 indicate a difference of diffusion between the water and methanol molecules in mixing condition, although not as high as for the pure systems. The concept of dynamical heterogeneity ${ }^{24-26}$ is about domains of different mobility within the same system, which is observed in jammed conditions typical of glasses ${ }^{28} \mathrm{In}$ glasses, these heterogeneities of the dynamics are induced by cooperative motions. In a micro-segregated system, we expect

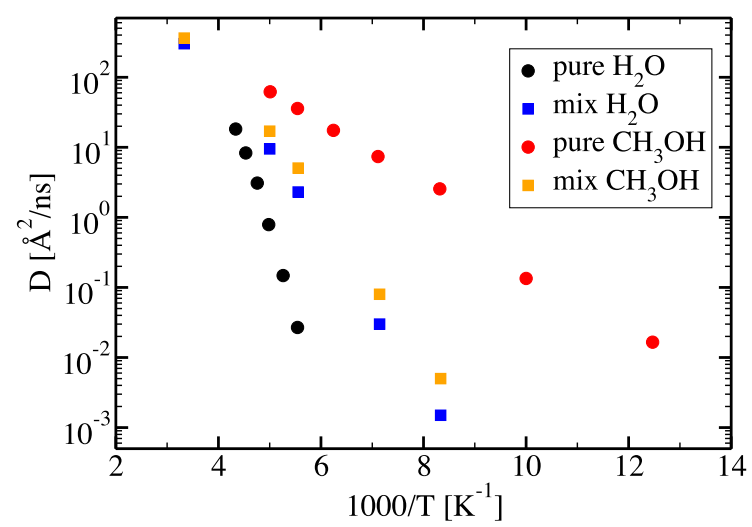

FIG. 7. Temperature dependence of the diffusion constant (in $\AA^{2} / \mathrm{ns}$ ) as a function of the inverse temperature, in neat liquids and equimolar mixture. a natural dynamical heterogeneity due to the discrepancy in diffusion between different species. In this way, Fig. 7 is an illustration of the dynamical heterogeneity, but even in a hot mixture under ambient conditions, which is a novel extension of this concept. Hence, the difference in species mobility offers a natural mobility segregation associated with that of the species segregation. We are not aware that this property has been mentioned previously in any other system. We conjecture here that all micro-segregated mixtures have inherent dynamical heterogeneity.

\section{DISCUSSION AND CONCLUSION}

This work shows the pronounced differences between water and methanol, both from static and dynamical points of view. In our classical models of both molecules, the hydrogen bonding interaction is described classically through the Coulomb interactions. Water oxygen has a partial charge of $q_{W} \approx 0.87 e$, while that of methanol is $q_{M} \approx 0.7 e$. This could be thought as a very small difference. On the other hand, methanol single hydroxyl group carries the methyl group, and moreover the $\mathrm{H}$-bonding is more linear (hence methanol forms chains). Water has a tetrahedral binding. Therefore, it would seem that it is more these geometric/topological factors that induce the large differences observed in this study, than the partial charges - or equivalently, the H-bond interactions themselves. From static point of view, the most important result of this study is concomitant decrease of the water concentration fluctuations with decreasing temperatures, while a broad cluster/domain pre-peak emerges around $k=0.2-1 \AA^{-1}$. This pre-peak has many internal structures, in some cases, which seems to be related to the chain-like water clusters and agglomerates of them, which are visible in the snapshots. Such clusters are equally visible at $\mathrm{T}=300 \mathrm{~K}$, but they contribute only to the $k=0$ concentration fluctuation peak. It is tempting to relate both features, and consider the same type of clusters as $\mathrm{CF}$ at high temperatures and $\mathrm{MH}$ at lower temperatures. This relationship is further reinforced by the behaviour of methanol, which shows fuzzy clusters at all temperatures and no domain-pre-peak, while maintaining the pure methanol chain-cluster pre-peak at $k=1-1.5 \AA^{-1}$. In other words, it is the statistical persistence of chain-like clusters of the respective hydroxyl groups which contribute to the two types of pre-peaks.

The effect of lowering the temperature seems to increase the persistence of domains, which evolve from mere concentration fluctuations to segregated cluster-domains. Our study provides some insight into the mechanisms behind $\mathrm{CF}$ and $\mathrm{MH}$ and their interrelations. Furthermore, we have proposed a new interpretation of the behaviour of the KBI in terms of the "renormalisation" of the interactions into clusters. In this interpretation, the KBI would partly reflect the domain-domain interactions in addition to reflecting particleparticle interactions. This interpretation is partly supported by the existence of domain/cluster pre-peaks between the $\mathrm{k}=0$ part and the main peak part of the structure factors. This interpretation would need to be refined by subsequent investigations. 
It is interesting to compare the pseudo-ideality found at lower temperature to the non-ideality that Mallamace et $a .^{32}$ find in their study of the relaxation process in supercooled aqueous methanol mixtures. Despite an apparent contradiction, both findings are perfectly compatible. Indeed, the appearance of "ideal" non-interacting clusters also implies intense interaction internal to such clusters, with associated relaxation processes. Similarly, the low temperature coarsening of the concentration fluctuations into separated water and methanol clusters, and their subsequent decrease, conspire against the scenario of a critical demixing, even hidden below the freezing line. ${ }^{31}$

Although we did not investigate the issue of a hidden second critical point for water, ${ }^{33,35}$ there is a possibility that many of the current findings in the literature ${ }^{36,43}$ could be related to the appearance of specific types of hydrogen bonded water clusters, which are absent in neat water. ${ }^{37,38,55}$ We did not investigate here the dynamical properties related to relaxations, such as dynamical structure factors ${ }^{27,28}$ or nonGaussian parameters, ${ }^{72,71}$ for example, which are a modern measure of glassiness. ${ }^{27,29} \mathrm{We}$ postpone such studies for future investigations.

Finally, this work provides a unified picture of the microheterogeneity in pure components and mixtures. Indeed, neat linear alcohols have a particular inhomogeneity in the form of linear chains formed by the hydroxyl groups. On the other hand, in our previous works on aqueous and nonaqueous mixture, we have underlined the micro-heterogeneity which appears under the form of segregated molecular domains. However, both forms of micro-heterogeneity have very different signature in the correlation functions. The first appears as a lowering of the second and third neighbour short range contributions to the correlation between the hydroxyl groups, whereas the second appears as long range oscillatory contributions to the same correlation functions. In the present work, we have seen that micro-heterogeneity of the first type could equally appear in mixing conditions, but at low temperatures. This way, micro-heterogeneity appears as a universal phenomena related to aggregate formations of different types, due to the same type of directional interactions, and with distinct signatures in the same correlation functions (that of the hydroxyl groups).

To conclude, through this study, we have shown some of the correspondences between concentration fluctuations and micro-segregation in relation to thermal agitation: lower temperatures are likely to select some types of concentration fluctuations and promote them into persistent domains. This is in relation with the sharp increase of the first peak of the correlation functions reported in Fig. 2, but also in second and third neighbour correlations. It is these contributions that contribute mostly to the structure factors at small $\mathrm{k}$ values, precisely in the pre-peak region. Micro-segregation is seen to have a diverse morphology, with chain-like water clusters in the present cases and in aqueous DMSO, ${ }^{42}$ and bulkier domains, as seen in aqueous mixtures with higher alcohols ${ }^{47}$ and polar molecules. ${ }^{48}$ In the first case, the signature in the correlation function is a lowering of the correlations in the short range part, while in the second case, it is a domain oscillatory behaviour in the long range part. Both features produce a domain pre-peak in the associated structure factors. In this work, we have shown that temperature can influence the formation of micro-heterogeneity in place of a demixing phase transition. We expect to pursue the analysis started here in other systems that we have previously studied from the point of view of micro-heterogeneity.

\section{ACKNOWLEDGMENTS}

This work has been supported in part by the Croatian Science Foundation for financial support under Project No. 4514 "Multi-scale description of meso-scale domain formation and destruction." M. Požar thanks the French Embassy in Croatia for financial support through "bourse du Gouvernement Français."

${ }^{1}$ S. Dixit, J. Crain, W. C. Poon, J. L. Finney, and A. K. Soper, Nature 416, 829 (2002).

${ }^{2}$ S. K. Allison, J. P. Fox, R. Hargreaves, and S. P. Bates, Phys. Rev. B 71, 024201 (2005).

${ }^{3}$ E. A. Ploetz and P. E. Smith, Phys. Chem. Chem. Phys. 13, 18154 (2014).

${ }^{4}$ E. A. Ploetz, N. Bentenitis, and P. E. Smith, Fluid Phase Equilib. 290, 43 (2010).

${ }^{5}$ D. Subramanian, D. A. Ivanov, I. K. Yudin, M. A. Anisimov, and J. V. Sengers, J. Chem. Eng. Data 56, 1238 (2011).

${ }^{6}$ S. Banerjee, J. Furtado, and B. Bagchi, J. Chem. Phys. 140, 194502 (2014).

${ }^{7}$ M. Požar, J. B. Seguier, J. Guerche, R. Mazighi, L. Zoranić, M. Mijakovic, N. Kežić-Lovrinčević, F. Sokolić, and A. Perera, Phys. Chem. Chem. Phys. 17, 9885 (2015).

${ }^{8}$ A. Perera, Pure Appl. Chem. 88, 189 (2016).

${ }^{9}$ S. Sarkar, S. Banerjee, S. Roy, R. Ghosh, P. Pratim Ray, and B. Bagchi, J. Chem. Sci. 127, 49 (2015).

${ }^{10}$ J. G. Kirkwood and F. Buff, J. Chem. Phys. 19, 774 (1950).

${ }^{11}$ E. Matteoli and L. Lepori, J. Chem. Phys. 80, 2856 (1984).

${ }^{12}$ A. Ben-Naim, J. Chem. Phys. 67, 4884 (1977).

${ }^{13}$ G. Z. Schneider, Z. Phys. Chem. N. F. 41, 327 (1964).

${ }^{14}$ A. G. Aizpiri, F. Monriy, C. Del Campo, R. G. Rubio, and M. Diaz Pena, Chem. Phys. 165, 31 (1992).

${ }^{15}$ A. Perera and F. Sokolic, J. Chem. Phys. 121(22), 11272 (2004).

${ }^{16}$ R. G. Peyrera, M. L. Asar, and M. A. Carignano, Chem. Phys. Lett. 507, 240 (2011).

${ }^{17}$ A. Pinke and P. Jedlovzky, J. Phys. Chem. B 116, 5977 (2012).

${ }^{18}$ L. Vrbka and P. Jungwirth, Phys. Rev. Lett. 95, 148501 (2005).

${ }^{19}$ M. Matsumoto, S. Saito, and I. Ohmine, Nature 416, 409 (2002).

${ }^{20}$ W. Kob and H. C. Anderson, Phys. Rev. E 51, 4626 (1995).

${ }^{21}$ A. P. Kerasidou, Y. Mauboussin, and V. Teboul, Chem. Phys. 450, 94 (2015).

${ }^{22}$ E. L. Gromnitskaya, O. V. Stal'gorova, O. F. Yagafarov, V. V. Brazhkin, A. G. Lyapin, and S. V. Popova, JETP Lett. 80, 697 (2004).

${ }^{23}$ Ó. Gálvez, B. Maté, B. Martín-Llorente, V. J. Herrero, and R. Escribano, J. Phys. Chem. A 113, 3321 (2009).

${ }^{24}$ M. D. Ediger, Annu. Rev. Phys. Chem. 51, 99 (2000).

${ }^{25}$ S. C. Glotzer, J. Non-Cryst. Solids 274, 342 (2000).

${ }^{26}$ H. C. Andersen, Proc. Natl. Acad. Sci. U. S. A. 102, 6686 (2005).

${ }^{27}$ L. Berthier and G. Biroli, Rev. Mod. Phys. 83, 587 (2011).

${ }^{28}$ L. Berthier, Physics 4, 42 (2011).

${ }^{29}$ V. Teboul and J. B. Accary, Phys. Rev. E 89, 012303 (2014).

${ }^{30}$ V. Teboul, M. Saiddine, and J.-M. Nunzi, Phys. Rev. Lett. 103, 265701 (2009).

${ }^{31}$ L. Dougan, R. Hargreaves, S. P. Bates, J. L. Finney, V. Réat, A. K. Soper, and J. Crain, J. Chem. Phys. 122, 174514 (2005).

${ }^{32}$ F. Mallamace, C. Corsaro, D. Mallamace, C. Vasi, S. Vasi, and H. E. Stanley, J. Chem. Phys. 144, 064506 (2016).

${ }^{33}$ P. H. P. Poole, F. Sciortino, U. Essmann, and H. E. Stanley, Nature 360, 324 (1992).

${ }^{34}$ D. Paschek and R. Ludwig, Angew. Chem., Int. Ed. 53, 11699 (2014).

${ }^{35} \mathrm{O}$. Mishima and H. E. Stanley, Nature 392, 164-168 (2998).

${ }^{36}$ Z. Zhao and C. A. Angell, Angew. Chem., Int. Ed. 55, 2474 (2016).

${ }^{37}$ L. Zoranić, F. Sokolić, and A. Perera, J. Chem. Phys. 127, 024502 (2007).

${ }^{38}$ A. Perera, F. Sokolic, and L. Zoranic, Phys. Rev. E 75, 060502(R) (2007).

${ }^{39}$ B. E. Warren, Phys. Rev. 44, 969 (1933). 
${ }^{40}$ R. Ludwig, Chem. Phys. Phys. Chem. 6, 1369 (2005).

${ }^{41}$ J.-H. Guo, Y. Luo, A. Auggustsson, S. Kashtanov, J.-E. Rubensson, D. K. Shuh, H. Agren, and J. Nordgren, Phys. Rev. Lett. 91, 157401-1 (2003).

${ }^{42}$ A. Perera and R. Mazighi, J. Chem. Phys. 143, 154502 (2015).

${ }^{43}$ K. I. Murata and H. Tanaka, Nat. Mater. 11, 436 (2012).

${ }^{44}$ J. P. Hansen and I. R. McDonald, Theory of Simple Liquids (Academic, London, 1986).

${ }^{45}$ A. Perera, B. Kežić, F. Sokolić, and L. Zoranić, in Molecular Dynamics, edited by L. Wang (InTech, Rijeka, 2012), Vol. 2.

${ }^{46}$ A. Perera, in Fluctuation Theory of Solutions: Applications in Chemistry, Chemical Engineering, and Biophysics, edited by P. E. Smith, J. P. O'Connell, and E. Matteoli (CRC Press Taylor and Francis, 2012), ISBN: 9781439899229.

${ }^{47}$ B. Kežić and A. Perera, J. Chem. Phys. 137, 014501 (2012).

${ }^{48}$ B. Kežić and A. Perera, J. Chem. Phys. 137, 134502 (2012).

${ }^{49}$ R. Lumry and S. Rajendar, Biopolymers 9, 1125 (1970).

${ }^{50}$ R. R. Krug, W. G. Hunter, and R. A. Grieger, Nature 261, 566 (1976).

${ }^{51}$ D. van der Spoel, E. Lindahl, B. Hess, G. Groenhof, A. E. Mark, and H. J. C. Berendsen, J. Comput. Chem. 26, 1701 (2005).

${ }^{52}$ J. M. Martínez and L. Martínez, J. Comput. Chem. 24(7), 819-825 (2003); L. Martínez, R. Andrade, E. G. Birgin, and J. M. Martínez, ibid. 30(13), 2157-2164 (2009).

${ }^{53}$ M. Tomšić, A. Jamnik, G. Fritz-Popovski, O. Glatter, and L. Vlcek, J. Phys. Chem. B 111, 1738 (2007).

${ }^{54}$ A. Perera, L. Zoranić, F. Sokolić, and R. Mazighi, J. Mol. Liq. 159, 52 (2011).

${ }^{55}$ A. Perera, Mol. Phys. 109, 2433 (2011).
${ }^{56}$ A. Perera and B. Kežić, Faraday Discuss. 167, 145 (2013).

${ }^{57}$ E. Oran Brigham, The Fast Fourier Transform (Prentice Hall, 1988).

${ }^{58}$ J. C. Berendsen, J. P. M. Postma, W. F. Von Gusteren, and J. Hermans, in Intermolecular Forces, edited by B. Pullman (Reidel, Dordrecht, 1981).

${ }^{59}$ W. L. Jorgensen, J. D. Madura, and C. J. Swenson, J. Am. Chem. Soc. 106, 6638 (1984); W. L. Jorgensen, J. Phys. Chem. 90, 1276 (1986).

${ }^{60}$ B. Chen, J. J. Potoff, and J. I. Siepmann, J. Phys. Chem. 105, 3093 (2001).

${ }^{61} \mathrm{C}$. A. Angell, in Glassy, Amorphous and Nano-Crystalline Materials, Hot Topics in Thermal Analysis and Calorimetry, edited by J. Sestàk (Springer Science+Business Media B.V, 2011), Vol. 8.

${ }^{62}$ M. Mezard and G. Parisi, J. Phys.: Condens. Matter 11, A157 (1999).

${ }^{63}$ G. Parisi, Proc. Natl. Acad. Sci. U. S. A. 103, 7948 (2006).

${ }^{64}$ L. Berthier and G. Biroli, "Glasses and aging: A statistical mechanics perspective," in Encyclopedia of Complexity and Systems Science, edited by E. Meyers and A. Roberts (Springer, New York, 2009).

${ }^{65}$ A. Sepulva, F. Stephen, and M. D. Ediger, J. Chem. Phys. 138, 12A517 (2013).

${ }^{66}$ M. P. Allen and D. J. Tildesley, Computer Simulation of Liquids (Oxford, 1987).

${ }^{67}$ A. A. H. Padua, M. F. Costa Gomes, and J. N. A. Canongia Lopes, Acc. Chem. Res. 40, 1087 (2007).

${ }^{68}$ Y. Wang and G. A. Voth, J. Am. Chem. Soc. 127, 12192 (2005).

${ }^{69}$ A. Perera et and R. Mazighi, J. Mol. Liq. 210, 243 (2015).

${ }^{70}$ B. Kežić and A. Perera, J. Chem. Phys. 135, 234104 (2011).

${ }^{71}$ M. S. Shell, P. G. Debenedetti, and F. H. Stillinger, J. Phys.: Condens. Matter 17, S4035 (2005).

${ }^{72}$ W. Kob, C. Donati, S. J. Plimpton, P. H. Poole, and S. C. Glotzer, Phys. Rev. Lett. 79, 2827 (1997). 